\title{
Diverse forms of HIV-1 among Burmese long-distance truck drivers imply their contribution to HIV-1 cross-border transmission
}

\author{
Yan-Heng Zhou ${ }^{1,4,5}$, Yue-Bo Liang ${ }^{2}$, Wei Pang ${ }^{1}$, Wei-Hong Qin ${ }^{2}$, Zhi-Hong Yao ${ }^{1,4}$, Xin Chen ${ }^{1,4}$, Chiyu Zhang ${ }^{3 *}$ \\ and Yong-Tang Zheng ${ }^{1 *}$
}

\begin{abstract}
Background: The China-Myanmar border is a particularly interesting region that has very high prevalence of and considerable diversity of HIV-1 recombinants. Due to the transient nature of their work, long-distance truck drivers (LDTDs) have a comparatively high potential to become infected with HIV-1 and further spread virus to other individuals in the area they travel within. In this study, we hypothesized that Burmese LDTDs crossing the China-Myanmar border frequently may potentially be involved in the cross-border transmission of HIV, and contribute to the extremely high prevalence of HIV-1 inter-subtype recombinants in this border region.
\end{abstract}

Methods: A molecular epidemiology study was conducted among 105 Burmese LDTDs between 2008 and 2010. HIV-1 genetic fragments including p17, pol, vif-vpr, vpr-env, and C2V3 were amplified and sequenced. The subtype characterization and HIV-1 transmission were determined by both phylogenetic and phylogeographic analyses.

Results: Diverse forms of HIV-1, including subtypes CRF01_AE (41.9\%), C (8.6\%), B (4.8\%), CRF02_AG (1.0\%), and inter-subtype recombinants (33.3\%), as well as dual infection (10.5\%), were detected among the tested LDTDs. Phylogeographic analyses based on pure subtype revealed that $77.8 \%$ Burmese LDTDs acquired HIV-1 infection in Yunnan, and the others in Myanmar. Both the C-related and CRF01_AE-related recombinants from these LDTDs appeared to have close genetic relationship with those from IDUs in Myanmar and Dehong.

Conclusions: Burmese LDTDs may contribute to HIV-1 transmission along the China-Myanmar border. The results may provide some new perspective for understanding the on-going generation and prevalence of HIV-1 recombinants in the border region.

Keywords: LDTDs, HIV-1 subtype, Recombination, Transmission, China-Myanmar border

\section{Background}

Myanmar has one of the highest rates of HIV-1 in southeast Asia, with CRF01_AE and B being the predominant subtypes among both injection drug users (IDUs) and heterosexual individuals in south and central Myanmar [1-3]. In northern Myanmar, CRF01_AE, B and $\mathrm{C}$-involved unique recombinant forms (URFs) were the most prevalent strains among IDUs [4]. China's

\footnotetext{
* Correspondence: zhangcy1999@ips.ac.cn; zhengyt@mail.kiz.ac.cn

${ }^{3}$ Pathogen Diagnostic Center, Institut Pasteur of Shanghai, Chinese Academy of Sciences, Shanghai 200025, China

'Key Laboratory of Animal Models and Human Disease Mechanisms of Chinese Academy of Sciences \& Yunnan Province, Kunming Institute of Zoology, Chinese Academy of Sciences, Kunming, Yunnan, China

Full list of author information is available at the end of the article
}

Yunnan province, which shares some 1977 kilometers of its border with Myanmar, has the highest number of HIV-1 infected individuals in China, accounting for $34.8 \%$ of national total cases as reported [5]. In Yunnan, multiple HIV-1 genotypes, including CRF01_AE, B, C, CRF07_BC, CRF08_BC, as well as various URFs, have been identified [6,7]. Within Yunnan, Dehong prefecture along the northern borders of Myanmar has considerably high HIV-1 prevalence among IDUs, and the proportion of HIV-1 recombinants reached $86.1 \%$, very similar to that of northern Myanmar [7-9]. Why such an extremely high prevalence of HIV-1 recombinants occurred in both sides of the China-Myanmar border remains unclear. 
Ruili city in Dehong prefecture is an important port linking China and Myanmar, serving as the major gateway for trade between both sides. Drugs (in particular heroin) from the infamous "Golden Triangle" are often illegally transferred to China via this port. Along the drug trafficking routes, HIV-1 has rapidly spread among IDUs. Unsurprisingly, Ruili became the first recorded site of HIV-1 outbreak in China [10]. About 45.2\% Burmese IDUs and $55.6 \%$ Chinese IDUs cross the China-Myanmar border each month to inject drugs, often with sharing needles and syringes [Dr. Lin Duo, personal communication]. This breadth of cross-border drug usage may partly explain the reason why both sides of the China-Myanmar border have similar HIV-1 epidemic and extremely high prevalence of HIV-1 recombinants.

Earlier studies supposed that HIV-1 expanded from Myanmar to Yunnan through these drug trafficking routes, but our recent phylogeographic analyses revealed that parental subtypes (e.g. B, C and CRF01_AE) of URFs_01BC circulating among Burmese IDUs were most likely transmitted from Dehong to Myanmar during 1990s [11]. Interestingly, the prevalence of CRF01_AE-related URFs was remarkably higher in northern Myanmar than in Dehong, Yunnan, China. IDUs may be a main factor fueling HIV-1 bidirectional transmission and generation of many URFs, but could not simply explain this substantial difference in HIV-1 recombination patterns between both sides of the border. Therefore, we were curious as to whether or not other groups aside from IDUs may also have contributed to the rapid mixing of HIV-1 subtypes $B$, $\mathrm{C}$ and CRF01_AE, and the generation of the extremely high prevalence of HIV-1 recombinants in the ChinaMyanmar border region.

Long-distance truck drivers (LDTDs) are at an increased risk of infection with HIV, since they often stay away from their hometowns for long periods of time, and therefore less frequently visit a local, monogamous sexual partner and more frequently use commercial sex workers (CSWs) and have multiple sexual partners $[12,13]$. A recent study showed that $5 \%$ of Burmese LDTDs crossing the China-Myanmar border had sex with occasional sexual partners during long-distance truck driving, and some LDTDs self-reported to inject drugs. Among this 5\% population, $7.1 \%$ reported that they did not use condoms when engaging in sexual activity. HIV-1 prevalence was reported to be as high as 3.5-8.8\% among Burmese LDTDs in general [14,15]. Whether these LDTDs were involved in the high prevalence of HIV-1 recombinants in the China-Myanmar border region needs to be determined.

Here, we conducted a molecular epidemiological study of HIV-1 among Burmese LDTDs, and investigated their phylogenetic relationship with strains circulating among IDUs in the China-Myanmar border region.

\section{Methods}

\section{Study population and specimens}

This study was conducted in accordance with the principles of the Declaration of Helsinki and relevant international regulations, and approved by the Ethics Committee of Kunming Institute of Zoology, Chinese Academy of Sciences (Approval ID: SWYX2008010). Under the support of Australia HIV/AIDS Asia Regional Program (HAARP) and Yunnan HAARP project, 105 blood specimens were collected from HIV-positive Burmese LDTDs entering and exiting Ruili port between 2008 and 2010, after they provided written informed consent. Demographic data of all participants were obtained via an interview. The plasma were separated and stored at $-70^{\circ} \mathrm{C}$ until use. All samples were serologically determined as HIV infection by an enzyme-linked immunosorbent assay (ELISA) kit (Wantai Biological Pharmacy Enterprise Co. Ltd, Beijing, China) and confirmed by another ELISA kit (Kehua bioengineering Co. Ltd, Shanghai, China). No HIV-2 infection was found among any of the studied participants.

\section{HIV-1 amplification, cloning and sequencing}

Five gene fragments including p17 (520 bp), pol (1273 bp), vif-vpr (702 bp), vpr-env (747 bp) and C2V3 (513 bp) were amplified from viral RNA by RT-nested PCR as described previously [4]. The amplicons were purified and sent to sequence directly without prior cloning (Invitrogen Trading, Shanghai, China). If direct sequencing failed due to the presence of numerous double peaks in the chromatogram, the amplicons were cloned into pMD19-T vectors (TaKaRa Biotechnology) and several clones were selected randomly for re-sequencing. Furthermore, if one amplified fragment of certain samples had a subtyping result different from all other four fragments, this fragment was also subjected to TA-clone and 20-25 colonies were selected randomly for sequencing.

\section{Phylogenetic analyses}

All obtained sequences were screened by the HIV BLAST tool to confirm lack of laboratory contamination and then were aligned with the HIV-1 reference strains obtained from the Los Alamos HIV database (http:// www.hiv.lanl.gov/content/sequence/NEWALIGN/align. html), using BioEdit 7.0.9 (http://www.mbio.ncsu.edu/ bioedit/bioedit.html) and MUSCLE (http://www.ebi.ac. $\mathrm{uk} /$ Tools $/ \mathrm{msa} / \mathrm{muscle} /$ ). Phylogenetic trees were constructed by the neighbor-joining (NJ) method with 1000 bootstrap replicates, using Kimura's two parameter model in MEGA 5.0 (http://www.megasoftware.net/). To detect HIV-1 recombinants, bootscan analysis was performed using SimPlot 3.5.1 (http://sray.med.som.jhmi.edu/SCRoft ware/simplot/). 
Phylogeographic analyses for CRF01_AE, B, C and URFs

To investigate the infection place of HIV-1 strains circulating among LDTDs, phylogeographic analyses were performed based on $p 17$ sequences using BEAST 1.6.2 $[16,17]$. Apart from $p 17$ sequences obtained in this study, all available sequences with known sample year from Myanmar, China, Thailand, Vietnam, and India were also included in the analyses. The time scaled maximum clade credibility (MCC) trees were constructed for CRF01_AE, subtype $\mathrm{B}, \mathrm{C}$ and URFs via the codon-based substitution model (SRD06), the uncorrelated log-normal relaxed clock model, and the constant population size. To ensure effective sample size (ESS) values $>200$, Markov Chain Monte Carlo (MCMC) chains for each subtype sequence set were run for 200 million generations. All trees were visualized using FigTree 1.3.1.

\section{Nucleotide sequence accession numbers}

All the sequences reported in this paper are available in GenBank under accession numbers from KC913597 to KC914092.

\section{Results}

Demographics of the study population

All 105 Burmese LDTDs were males with an average age of 34.3 (range: $20-56$ years). More than half of participants (58.1\%) came from Mandalay, a city in central Myanmar. Sixty five participants (61.9\%) self-reported the potential routes for HIV-1 infection including 59 (56.2\%) via sexual contact, two $(1.9 \%)$ via blood transfusion, two $(1.9 \%)$ via sexual contact and/or transfusions, and two (1.9\%) via sexual and/or injection drug use (IDU). None self-reported that IDU was the only risk behavior (see Additional file 1).

\section{Subtype characterization of HIV-1 strains circulating among Burmese LDTDs}

From 105 samples, we obtained 100 (95.2\%) p17, 86 (81.9\%) pol, 97 (92.4\%) vif-vpr, 85 (81.0\%) vpr-env and 90 (85.7\%) C2V3 sequences. Because of an overlap of $156 \mathrm{nt}$ between $v i f-v p r$ and $v p r$-env sequences, they were spliced into a $1328 \mathrm{nt}$ sequence named vif-env when both were available for the same sample. As a result, 80 vif-env sequences were obtained (see Additional file 2).

Based on four fragments $p 17$, pol, vif-env and $C 2 V 3$, we analyzed the subtype characterization of HIV-1 among Burmese LDTDs by constructing neighbor joining (NJ) phylogenetic trees and performing bootscan analyses. The results showed that CRF01_AE was the most dominant subtype among Burmese LDTDs, accounting for 53.5\% (46/86 in $p o l)$ to $60.0 \%$ (60/100 in $p 17)$, based on different fragments. Summarizing the results of four fragments, CRF01_AE had a prevalence rate of $41.9 \%$. Only nine C (8.6\%), five B (4.8\%), and one CRF02_AG (1.0\%) were identified among this cohort (Figure 1A). Interestingly, a total of 35 inter-subtype recombinants were identified, accounting for 33.3\%. BLAST and Simplot analyses showed that most recombinants did not share completely same recombination patterns with previously reported CRFs and URFs, suggesting that they were new URFs. The recombinants were comprised of CRF01_AE/C (37.1\%), CRF01_AE/B (25.7\%), B/C (22.9\%), and CRF01_AE/B/C (14.3\%) (Figure 1B). In details, 15 and 20 recombinants were detected based on pol and vif-env regions, respectively. Intriguingly, the strain from 08mLDTD008 has a pol fragment like CRF08_BC, but a vif-env fragment different from CRF08_BC. In addition, strains from 09mLDTD023, 09mLDTD028 and 09mLDTD029 seemed to share the same chimeric map with CRF07_BC in vif-env fragment, but different map in other fragments (see Additional files 2,3,4). In particular, 09mLDTD023 also shared two of three breakpoints with CRF07_BC in pol fragment (Additional file 3). These findings suggested that $08 \mathrm{~mL}$ DTD008, 09mLDTD023, 09mLDTD028 and 09mLDTD 029 might originate from the second-generation recombination of CRF07_BC or CRF08_BC with other subtypes.

High prevalence of HIV-1 dual infection among LDTDs

Among the studied Burmese LDTDs, 10.5\% were found to be dually infected with distinct subtypes or URFs. A majority $(72.7 \%)$ of them were dually infected with CRF01_AE \& C, followed by CRF01_AE \& B (9.1\%) (Figure 1C). Interestingly, 08mLDTD003 was found to be infected with subtype $\mathrm{C}$ and a $\mathrm{B} / \mathrm{C}$ recombinant, and 09mLDTD011 was triply infected with CRF01_AE and two different CRF01_AE/B recombinants. BLAST and bootscan analyses showed that the $\mathrm{B} / \mathrm{C}$ and two CRF01_AE/B recombinants mentioned above were different from any other URFs identified in this and previous studies. Further phylogenetic analyses showed that the subtype $\mathrm{C}$ region of the $\mathrm{B} / \mathrm{C}$ recombinant from 08mLDTD003 clustered closely with the subtype $C$ sequences from the same individual (Figure 2A). Similarly, the CRF01_AE parts of two CRF01_AE/B recombinants from 09mLDTD011 clustered closely with the CRF01_AE sequences from the same individual (bootstrap values $>80$ ) (Figure $2 \mathrm{~B}$ and $\mathrm{C}$ ). Furthermore, the subtype B parts shared by the two CRF01_AE/B recombinants from 09mLDTD011 clustered together closely, suggesting a common origin of the subtype B part. Taken together, these findings suggested that the three recombinants identified in the two co-infected individuals most likely originated from recombination events recently occurring in these two individuals, rather than through direct transmission from other individuals.

\section{Origin of HIV-1 among LDTDs}

To investigate where these LDTDs might have been infected by HIV-1, phylogeographic analyses were performed based on $p 17$ sequences of pure subtypes. The 


\section{(A)}

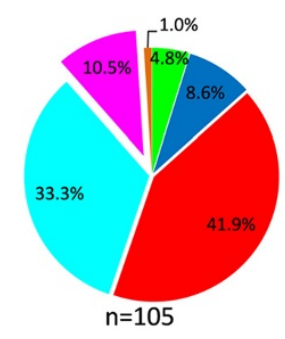

$\| \mathrm{B} \equiv \mathrm{C} \equiv$ CRF01_AE $\equiv$ Recombinants $\equiv$ Dual infection $\equiv$ 02_AG

(B)

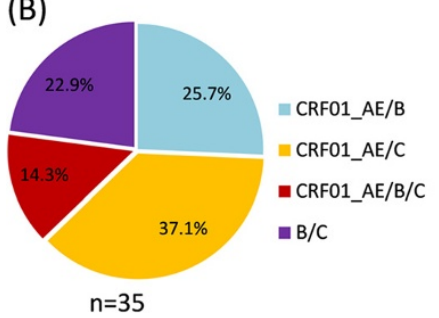

(C)

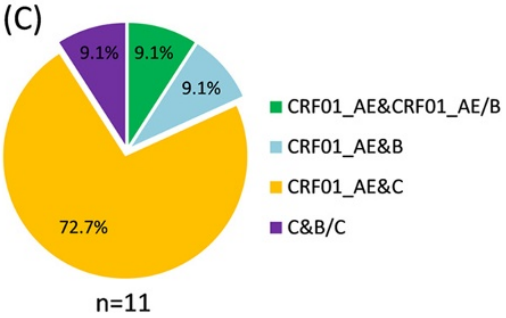

Figure 1 Molecular epidemiological characterization of HIV-1 circulating among Burmese LDTDs. (A) the proportion of HIV-1 subtypes, CRFs, recombinants and dual infection (B) the proportion of various recombinants (C) the proportion of dual infection (DI) patterns.

results showed that all five subtype B (100\%), six subtype C $(85.7 \%, 6 / 7)$ and 31 CRF01_AE $(73.8 \%, 31 / 42)$ sequences from the Burmese LDTDs had a geographic origin in Yunnan (Figure 3). Only one subtype $C$ and 9 CRF01_AE sequences from Burmese LDTDs were found to originate in Myanmar, suggesting that Burmese LDTDs were infected with HIV-1 in either Yunnan or Myanmar.

In order to further investigate the potential role of LDTDs in HIV-1 transmission, we also conducted phylogeographic analyses based on $p 17$ regions of URFs from LDTDs, IDUs, and CSWs in Myanmar and IDUs in Dehong, China. Two MCC trees were constructed for URFs with $p 17$ regions of subtype C-origin (named as the C-related URFs) and CRF01_AE-origin (named as the CRF01_AE-related URFs) (Figure 4). In the tree of subtype C-related URFs, Burmese LDTDs sequences, regardless of pure subtype $C$ sequences and the $C$ related URFs, clustered together and formed four clusters (Figure 4A). Two clusters including 11 Burmese LDTDs sequences and one cluster including six Burmese LDTDs sequences appeared to originate from Dehong and Dehong IDUs, respectively. The forth cluster including five Burmese LDTDs sequences appeared to have an origin from Burmese IDUs. In the tree of CRF01_AErelated URFs, majority of Burmese LDTDs sequences also clustered together. For these URFs, 5 (31.3\%) originated from Dehong, 6 (40.0\%) from Myanmar or Burmese IDUs, and one from Thailand. Interestingly, we found that sporadic sequences from Burmese IDUs are derived from Burmese LDTDs, suggesting that Burmese LDTDs spread HIV-1 to IDUs.

\section{Discussion}

Population movement is commonly identified as a key factor associated with transmission of human viruses, especially HIV-1 [18]. LDTDs are a particular group with high potential to be infected with HIV-1 and further spread the virus to other individuals due to the long distances they travel, the long duration of staying away from hometowns, frequent use of CSWs, and some of them also being IDUs. Several previous studies have reported the association of the high risk behaviors of LDTDs with HIV-1 infection [15,19]. However, there is less paper to characterize the molecular epidemiology of HIV-1 among LDTDs.

The China-Myanmar border region is a particularly interesting area that serves as a key channel for illegal drug trafficking from the "Golden triangle" to other countries/regions and has very high prevalence of HIV-1 and other viruses (e.g. HCV and HBV) $[4,9,20]$. In particular, over $86 \%$ HIV-1 strains circulating among IDUs in this region belong to URFs, which raises a question why such high proportion of HIV-1 recombinants occurred among them in this region. Furthermore, CRF01_AE-related URFs were detected among $45.6 \%$ of recombinants in northern Myanmar, significantly higher than $6.6 \%$ in its counterpart side (i.e. Dehong, Yunnan province of China) $[4,9]$. IDUs were postulated as a main population responsible for the generation of so many URFs since they often injected drugs together across the border and shared needles/syringes. However, the behaviors of IDUs do not explain why such high proportion $(45.6 \%)$ of CRF01_AE-related URFs occurs in northern Myanmar, implying the presence of other high-risk population 


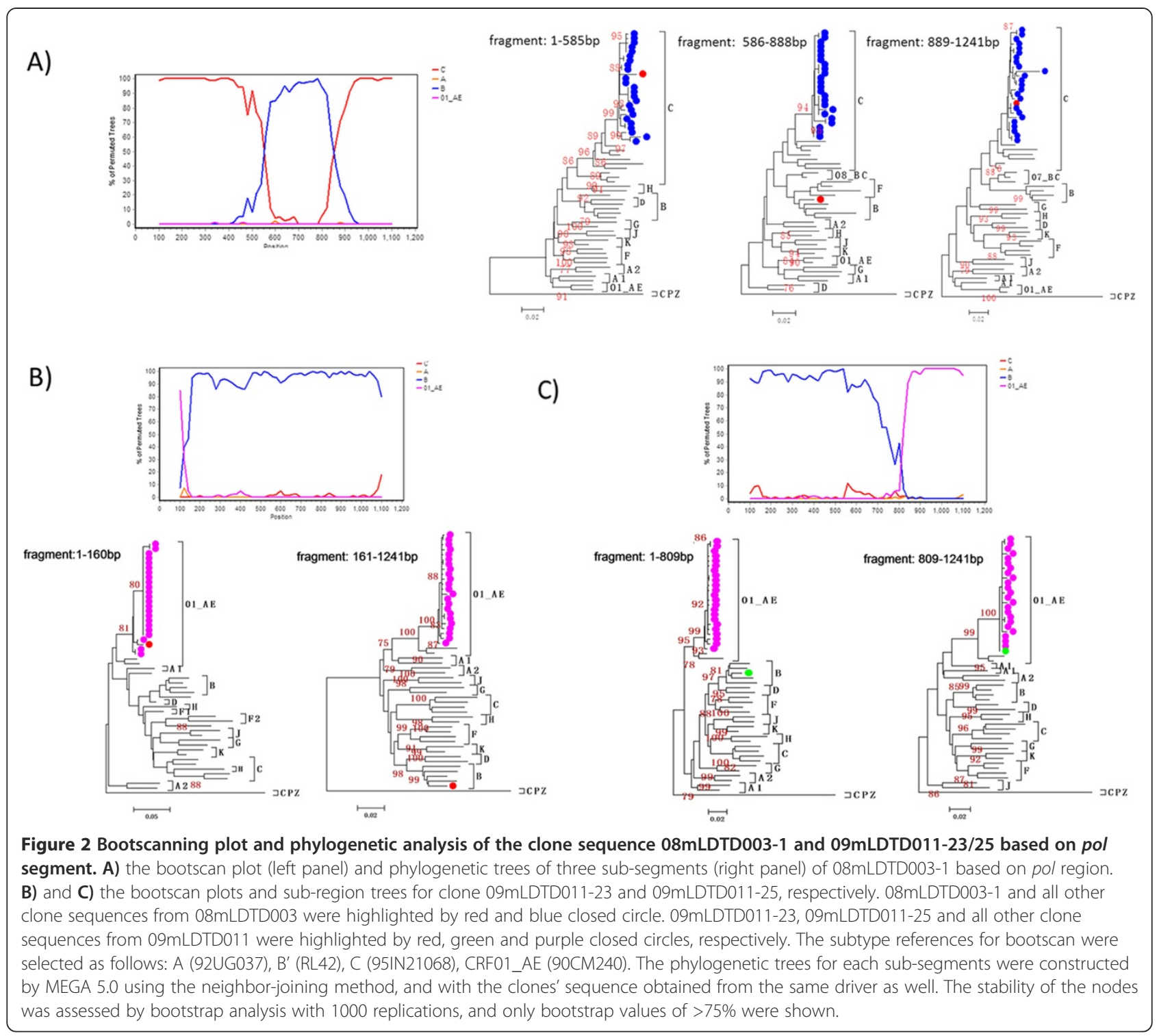

responsible for this difference. In this study, we collected the samples from 105 Burmese LDTDs who drive between Mandalay, Myanmar and Ruili, China, and performed a molecular epidemiological investigation of HIV-1 among this cohort. The results showed that diverse forms of HIV-1, including subtypes CRF01_AE (41.9\%), C (8.6\%), B (4.8\%), CRF02_AG (1.0\%), and some inter-subtype recombinants (33.3\%), were circulating among these LDTDs.

HIV-1 CRF01_AE is the most predominant subtype circulating among sexually acquired cases, where the prevalence of inter-subtype recombinants is relatively very low [6]. By contrast, inter-subtype recombinants are the most predominant forms circulating among IDUs, who have a very low prevalence of CRF01_AE $[4,6]$. Our results showed that CRF01_AE and inter-subtype recombinants were the two most predominant forms of HIV-1 among Burmese LDTDs (Figure 1 and Additional file 2).
Comparison showed that CRF01_AE prevalence among Burmese LDTDs was in the middle between heterosexuals (77.1\% in Dehong and 60.5\% in Mandalay) and IDUs (1.3\% in Myitkyina 19.0\% in Mandalay and 1\% in Dehong), implying that Burmese LDTDs might contribute to the epidemiological link between heterosexuals and IDUs. Among 65 LDTDs with available information on infection routes, 59 (90.8\%) self-reported that they were infected via sexual contact and two (3.1\%) via sexual contact and/or IDU. This provides realistic evidence to support the role of LDTDs in linking heterosexuals and IDUs. On the other hand, the prevalence of recombinants among Burmese LDTDs was also somewhere in between both sides of the China-Myanmar border $(71.4 \%$ and $87.3 \%$ in Dehong and Myitkyina IDUs, respectively) and Mandalay (28.6\% and 7.9\% among Mandalay IDUs and sexual intercourse group, respectively) (Figure 5). These suggest that 


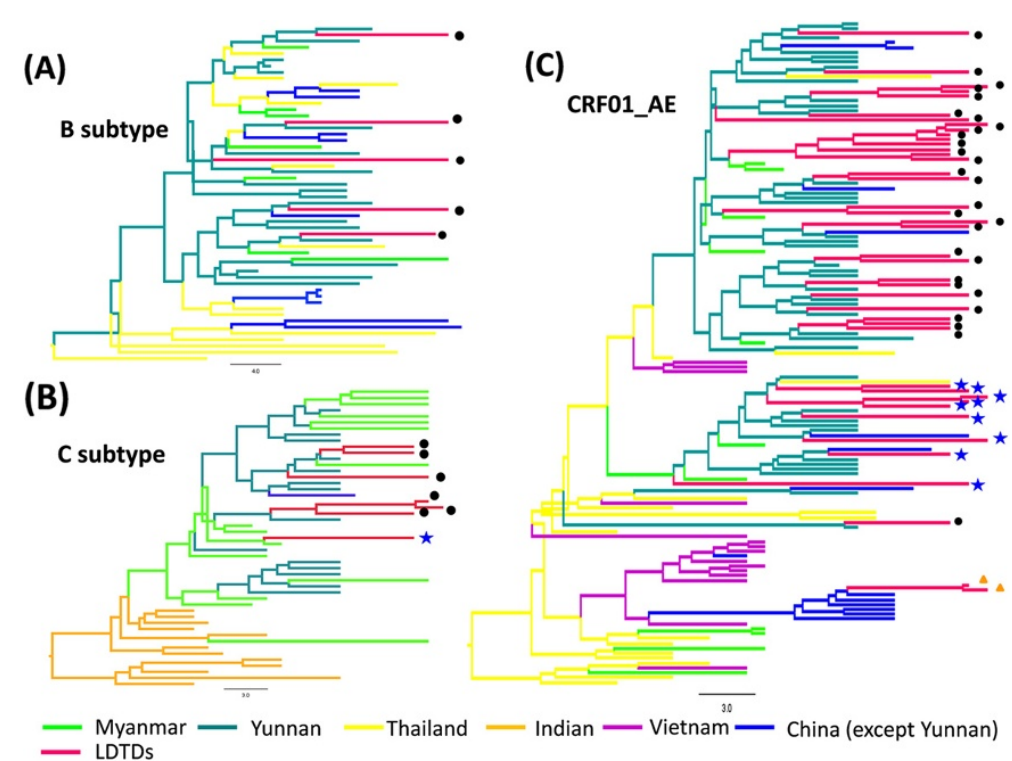

Figure 3 Bayesian maximum clade credibility (MCC) trees of HIV-1 strains from Burmese LDTDs. HIV-1 subtype B (A), C (B) and CRF01_AE (C). The trees were reconstructed based on $p 17$ sequences. The tree branches were signed by different color according to their respective geographical location. The black solid circle and the blue solid star indicated the sequences from Burmese LDTDs having geographic origin in Yunnan and Myanmar, respectively.
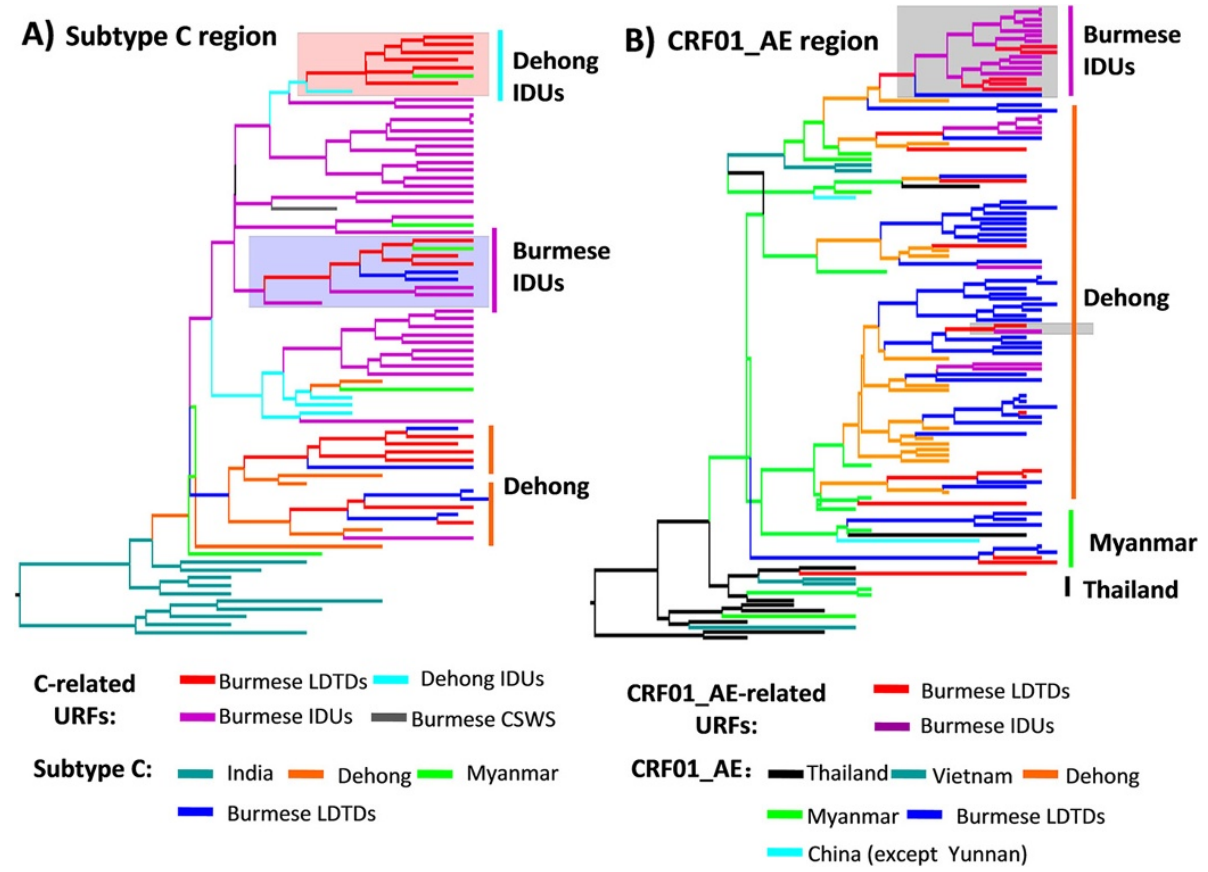

Figure 4 Bayesian maximum clade credibility (MCC) trees of HIV-1 URFs from Burmese LDTDs. p17 fragments of HIV-1 URFs belong to subtype C (A) or CRF01_AE (B). Trees were also reconstructed based on p17 sequences. The reference sequences were comprised of pure subtype and the corresponding parts of the URFs from Myanmar and Dehong (download from the ref 2, 3, 4, 8). The tree branches were signed by different color according to their respective geographical location. The pink shade and lilac shade in the part A highlighted C-related URFs of Burmese LDTDs originating from Dehong IDUs and Myanmar IDUs, respectively. In the part B, the grey shade highlighted the relationship of CRF01_AE-related URFs between Myanmar IDUs and LDTDs. 


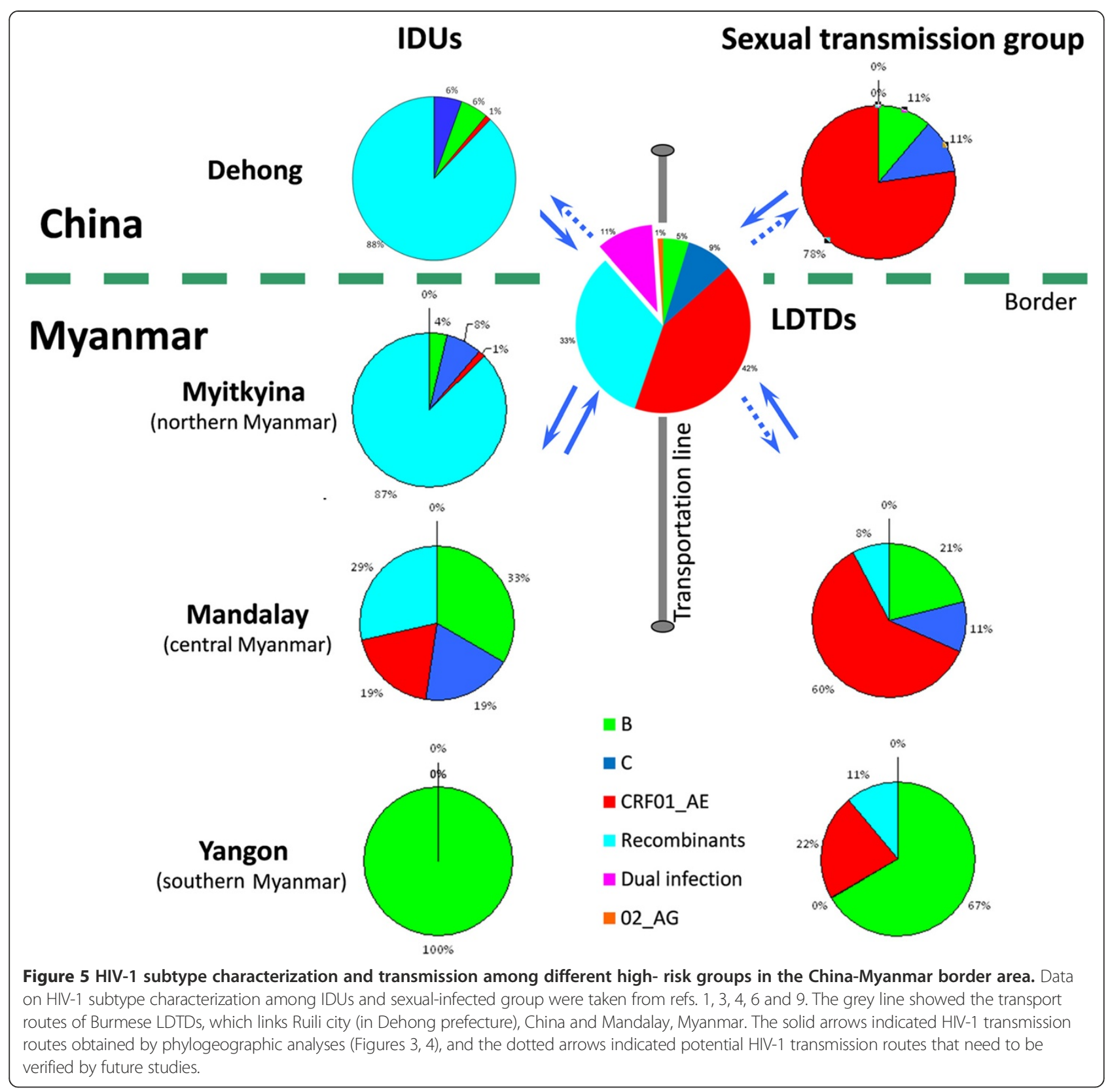

Burmese LDTDs may also have contributed to HIV-1 transmission between China and Myanmar. Taking these results together, we speculated Burmese LDTDs might mediate the cross-border transmission of CRF01_AE from heterosexuals to IDUs.

Phylogeographic analyses of the pure subtypes showed that majority $(77.8 \%, 42 / 54)$ of Burmese LDTDs were infected with HIV-1 (five subtype B, six subtype $C$ and 31 CRF01_AE) in Yunnan, and the others were infected in Myanmar (one subtype C and 9 CRF01_AE) (Figure 3). Likewise, MCC trees based on $p 17$ regions derived from the URFs of LDTDs also supported that HIV-1 C and CRF01_AE in LDTDs have multiple origins including
Dehong and Myanmar (Figure 4). On a broader scale, no matter where Burmese LDTDs acquired their infection, they have high potential to further spread the viruses to their wives and/or sexual partners in Myanmar and Yunnan. Therefore, Burmese LDTDs can mediate bidirectional transmission of HIV-1 between heterosexuals and IDUs and between China and Myanmar. This bi-directional transmission pattern of HIV-1 will facilitate the mixing of different HIV-1 forms among different high risk groups, and accelerate the generation of new recombinants, as observed among IDUs in the China-Myanmar border region. This concept was further supported by the detection of all CRF01_AE, B and C involved 
recombination forms (e.g. B/C, CRF01/B, CRF01/C, and $\mathrm{CRF} 01 / \mathrm{B} / \mathrm{C}$ ) among Burmese LDTDs (Figure 1B). One major limitation is that because the samples from female sexual workers (FSWs) in the China-Myanmar border region were unavailable in this study, we were unable to obtain HIV-1 subtype characterization in this cohort, and could not find the direct evidence for the transmission association between LDTDs and FSWs. Hence we just further determined their role in HIV-1 cross-border transmission and their association with IDUs via phylogeographic analyses. To understand HIV genetic diversity and transmission complexity in the China-Myanmar border region, more concern should be paid to female sexual workers in future.

Dual infection is an essential condition for the generation of HIV-1 recombinants [21,22]. Currently, intersubtype recombinants are responsible for about $11.0 \%$ HIV-1 infection throughout the Asia (http://www.hiv. lanl.gov/), and dual/triple infections have been detected in Thailand and Taiwan, where multiple subtypes are co-existing [23-25]. In the China-Myanmar border area, 86.1-87.9\% HIV-1 infected IDUs carried recombinants $[4,9]$. Such high prevalence of recombinants implies a very frequent occurrence of dual infection. To date, however, no dual infection case was reported in this region. We found that 10.5\% Burmese LDTDs were dually infected with various subtypes, implying frequent exposure of LDTDs to HIV-1. Among them, dual infection with CRF01_AE and C accounted for 72.7\% (Figure 1C), which not only provided an explanation for why CRF01_AE/C recombinants were the most predominant recombination form (37.1\%) among Burmese LDTDs, but also predicted an on-going generation of more new CRF01_AE/C recombinants among this population. In addition, CRF01_AE was involved in $90.9 \%$ cases of dual infection. These, together with the fact of high prevalence of CRF01_AE (41.9\%), support the close association of Burmese LDTDs with sexual transmission group.

Interestingly, two cases were found to be dual infection with certain pure subtype and its self-derived recombinants: one with CRF01_AE and CRF01_AE/B recombinants and the other with subtype $C$ and $B / C$ recombinant (Figure 2). These might represent a real-time observation on new recombinant generation. Because only the samples with sequences containing double peaks in Sanger sequencing were subjected to TA-cloning and in general, only 3-25 clones for each sample were selected for sequencing, this may lead to an underestimation of prevalence of dual infection among this cohort. The real dual infection rate may be higher when more samples are subjected to TA-cloning and sequencing, or ultra-deep sequencing, which is a robust tool to found lowfrequency variants.

\section{Conclusions}

This study firstly represented the molecular epidemiological characterization of HIV-1 among Burmese LDTDs. High prevalence of HIV-1 CRF01_AE and inter-subtype recombinants, as well as dual infection, were detected among them. Phylogeographic analyses revealed that Burmese LDTDs may have made contribution in HIV-1 transmission between the heterosexuals and IDUs and between China and Myanmar in general. These findings may provide new insight into understanding of the extensive and complex of HIV-1 recombination in the China-Myanmar border area (Figure 5). In a broader sense, our results also provide new information relevant to policy-making decisions regarding the control of HIV/AIDS in southeast Asia.

\section{Additional files}

Additional file 1: Characteristics and subtypes information of 105 Burmese LDTDs.

Additional file 2: Summary of subtype characterization of four gene fragments among 105 Burmese LDTDs.

Additional file 3: Bootscanning plots of pol fragments of HIV-1 inter-subtype recombinants among Burmese LDTDs. Recombination was determined using bootscan analyses (SimPlot 3.5.1 software). The HIV-1 subtype references used in bootscan analyses include subtypes A (92UG037), B' (RL42), C (95IN21068), and CRF01_AE (90CM240).

Additional file 4: Bootscanning plots of vif-env fragments of HIV-1 inter-subtype recombinants among Burmese LDTDs. Because some recombinants (e.g. B/C recombinants) shared identical breakpoints, only one representative bootscanning plot was shown. For the details of reference, please see Additional file 3 .

\section{Competing interests}

The authors declare there are no conflict interests.

\section{Authors' contributions}

YTZ and CZ conceived and designed the experiments; YBL and WHQ collected the samples; YHZ, WP and ZHY performed the experiments; YHZ, CZ, WP and $X C$ participated in the analyses of the data; $Y H Z, C Z$ and $Y T Z$ wrote and edited the manuscript. All authors read and approved the final manuscript.

\section{Acknowledgements}

The authors gratefully thank the participants in this study and the staff of the Australia HIV/AIDS Asia Regional Program (HAARP) and the Yunnan HAARP project helping collecting samples. We also thank Dr. Andrew Willden for editing the manuscript. This work was supported in part by grants from the NSFC (81271892, U1302224, U1202228, 81172876), Key Scientific and Technological Program of China (2012ZX10001-006, 2012ZX10001-007), National Basic Research Program of China (2012CBA01305), the Chinese Academy of Sciences (KSCX2-EW-R-13). The funders had no role in study design, data collection and analysis, decision to publish, or preparation of the manuscript.

\section{Author details}

${ }^{1}$ Key Laboratory of Animal Models and Human Disease Mechanisms of Chinese Academy of Sciences \& Yunnan Province, Kunming Institute of Zoology, Chinese Academy of Sciences, Kunming, Yunnan, China. ${ }^{2}$ Yunnan International Travel Healthcare Center, Kunming, Yunnan 650200, China. ${ }^{3}$ Pathogen Diagnostic Center, Institut Pasteur of Shanghai, Chinese Academy of Sciences, Shanghai 200025, China. ${ }^{4}$ University of the Chinese Academy of Sciences, Beijing 100049, China. ${ }^{5}$ Shaanxi Engineering \& Technological Research Center for Conversation \& Utilization of Regional Biological Resources, College of Life Sciences, Yan'an University, Yan'an, Shaanxi 716000, China. 
Received: 19 February 2014 Accepted: 19 August 2014

Published: 26 August 2014

\section{References}

1. Kusagawa S, Sato H, Watanabe S, Nohtomi K, Kato K, Shino T, Thwe M, OO KY, Lwin S, Mra R, Kywe B, Yamazaki S, Takebe Y: Genetic and serologic characterization of HIV type 1 prevailing in Myanmar (Burma). AIDS Res Hum Retroviruses 1998, 14:1379-1385.

2. Motomura K, Kusagawa S, Kato K, Nohtomi K, Lwin HH, Tun KM, Thwe M, Oo KY, Lwin S, Kyaw O, Zaw M, Nagai Y, Takebe Y: Emergence of new forms of human immunodeficiency virus type 1 intersubtype recombinants in central Myanmar. AIDS Res Hum Retroviruses 2000, 16:1831-1843.

3. Takebe Y, Motomura K, Tatsumi M, Lwin HH, Zaw M, Kusagawa S: High prevalence of diverse forms of HIV-1 intersubtype recombinants in Central Myanmar: geographical hot spot of extensive recombination. AIDS 2003, 17:2077-2087.

4. Pang W, Zhang C, Duo L, Zhou YH, Yao ZH, Liu FL, Li H, Tu YQ, Zheng YT: Extensive and complex HIV-1 recombination between $\mathrm{B}, \mathrm{C}$ and CRF01_AE among IDUs in southeast Asia. AIDS 2012, 26:1121-1129.

5. Jia M, Luo H, Ma Y, Wang N, Smith K, Mei J, Lu R, Lu J, Fu L, Zhang Q, Wu Z, Lu L: The HIV epidemic in Yunnan Province, China, 1989-2007. J Acquir Immune Defic Syndr 2010, 53(Suppl 1):S34-S40.

6. Zhang Y, Lu L, Ba L, Liu L, Yang L, Jia M, Wang H, Fang Q, Shi Y, Yan W, Chang G, Zhang L, Ho DD, Chen Z: Dominance of HIV-1 subtype CRF01_AE in sexually acquired cases leads to a new epidemic in Yunnan province of China. PLOS Med 2006, 3:e443.

7. Li L, Chen L, Yang S, Li T, Li J, Liu Y, Jia L, Yang B, Bao Z, Li H, Wang X, Zhuang D, Liu S, Li J: Recombination form and epidemiology of HIV-1 unique recombinant strains identified in Yunnan, China. PLoS One 2012, 7:e46777.

8. Yang $R$, Xia X, Kusagawa S, Zhang C, Ben K, Takebe Y: On-going generation of multiple forms of HIV-1 intersubtype recombinants in the Yunnan Province of China. AIDS 2002, 16:1401-1407.

9. Han X, An M, Zhao B, Duan S, Yang S, Xu J, Zhang M, McGoogan JM, Takebe Y, Shang $\mathrm{H}$ : High prevalence of HIV-1 intersubtype $\mathrm{B}^{\prime} / \mathrm{C}$ recombinants among injecting drug users in Dehong, China. PLoS One 2013, 8:e65337.

10. du Guerny J, Hsu L, Hong C: Population Movement and HIV/AIDS: the Case of Ruili, Yunnan, China. In UNDP Southeast Asia HIV and Development Programme; 2003 [Available at: http://www.hivdevelopment.org/]

11. Liu J, Jia Y, Xu Q, Zheng YT, Zhang C: Phylodynamics of HIV-1 unique recombinant forms in China-Myanmar border: implication for HIV-1 transmission to Myanmar from Dehong, China. Infect Genet Evol 2012, 12:1944-1948.

12. Manjunath JV, Thappa DM, Jaisankar TJ: Sexually transmitted diseases and sexual lifestyles of long-distance truck drivers: a clinico-epidemiologic study in south India. Int J STD AIDS 2002, 13:612-617.

13. Rao KS, Pilli RD, Rao AS, Chalam PS: Sexual lifestyle of long distance lorry drivers in India: questionnaire survey. BMJ 1999, 318:162-163.

14. Liang YB, Li YP, Wang CM, Li XY, Yang RQ, Su H, Wang GL, Wang ZJ: An analysis of HIV detection results among the Burmese truck drivers entering and exiting Ruili port. J Chin AIDS/STD 2010, 16:372-374.

15. Deng L, Zhang MY, Wang GL, Li SH, Xue HM, Duo L: Analysis on the effective elements relating Myanmar cross-border long-distance truck driver cross-border and risky behavior. Soft Sci Health 2011, 25:835-837.

16. Drummond AJ, Rambaut A: BEAST: Bayesian evolutionary analysis by sampling trees. BMC Evol Biol 2007, 7:214.

17. Lemey P, Rambaut A, Drummond AJ, Suchard MA: Bayesian phylogeography finds its roots. PLoS Comput Bio 2009, 5:e1000520.

18. Deane KD, Parkhurst JO, Johnston D: Linking migration, mobility and HIV. Trop Med Int Health 2010, 15:1458-1463.

19. Sanjeev K, S K G, S K B: A study of knowledge, sexual behaviour and practices regarding HIV/AIDS among long distance truck drivers. Ind J Public Health 2009, 53:243-245.

20. Zhou YH, Liu FL, Yao ZH, Duo L, Li H, Sun Y, Zheng YT: Comparison of HIV-, HBV-HCV- and co-infection prevalence between Chinese and Burmese intravenous drug users of the China-Myanmar border region. PLoS One 2011, 6:e16349.

21. Nájera R, Delgado E, Pérez-Alvarez L, Thomson MM: Genetic recombination and its role in the development of the HIV-1 pandemic. AIDS 2002, 16(Suppl 4):S3-S16.
22. Templeton AR, Kramer MG, Jarvis J, Kowalski J, Gange S, Schneider MF, Shao Q, Zhang GW, Yeh MF, Tsai HL, Zhang H, Markham RB: Multiple-infection and recombination in HIV-1 within a longitudinal cohort of women. Retrovirology 2009, 6:54

23. Hu DJ, Subbarao S, Vanichseni S, Mock PA, Ramos A, Nguyen L, Chaowanachan T, Griensven F, Choopanya K, Mastro TD, Tappero JW: Frequency of HIV-1 dual subtype infections, including intersubtype superinfections, among injection drug users in Bangkok, Thailand. AIDS 2005, 19:303-308

24. Chen YJ, Lee CM, Chen M, Chuang SY, Liu HF, Wong WW, Lin YH, Tsai HC, Wang JH, Chen YM: Molecular epidemiology of HIV-1 infection in Taiwan from 2005 to 2008: further spread of CRF07_BC and emergence of CRF07_BC/subtype B dual infection. J Acquir Immune Defic Syndr 2012, 59:438-446.

25. van der Kuyl AC, Zorgdrager F, Jurriaans S, Back NK, Prins JM, Brinkman K, van Eeden A, Bakker M, Cornelissen M: Incidence of human immunodeficiency virus type 1 dual infections in Amsterdam, The Netherlands, during 2003-2007. Clin Infect Dis 2009, 48:973-978.

doi:10.1186/1471-2334-14-463

Cite this article as: Zhou et al:: Diverse forms of HIV-1 among Burmese long-distance truck drivers imply their contribution to HIV-1 cross-border transmission. BMC Infectious Diseases 2014 14:463.

\section{Submit your next manuscript to BioMed Central and take full advantage of:}

- Convenient online submission

- Thorough peer review

- No space constraints or color figure charges

- Immediate publication on acceptance

- Inclusion in PubMed, CAS, Scopus and Google Scholar

- Research which is freely available for redistribution 